\title{
THE EXTENSION OF MONTGOMERY IDENTITY VIA FINK IDENTITY WITH APPLICATIONS
}

\author{
A. AGLIĆ ALJINOVIĆ, J. PEČARIĆ, AND A. VUKELIĆ
}

Received 30 January 2004

The new extension of the weighted Montgomery identity is given by using Fink identity and is used to obtain some Ostrowski-type inequalities and estimations of the difference of two integral means.

\section{Introduction}

The following Ostrowski inequality is well known [10]:

$$
\left|f(x)-\frac{1}{b-a} \int_{a}^{b} f(t) d t\right| \leq\left[\frac{1}{4}+\frac{(x-(a+b) / 2)^{2}}{(b-a)^{2}}\right](b-a) L, \quad x \in[a, b],
$$

where $f:[a, b] \rightarrow \mathbb{R}$ is a differentiable function such that $\left|f^{\prime}(x)\right| \leq L$, for every $x \in[a, b]$.

The Ostrowski inequality has been generalized over the last years in a number of ways. Milovanović and Pečarić [8] and Fink [6] have considered generalizations of (1.1) in the form

$$
\left|\frac{1}{n}\left(f(x)+\sum_{k=1}^{n-1} F_{k}(x)\right)-\frac{1}{b-a} \int_{a}^{b} f(t) d t\right| \leq K(n, p, x)\left\|f^{(n)}\right\|_{p}
$$

which is obtained from the identity

$$
\frac{1}{n}\left(f(x)+\sum_{k=1}^{n-1} F_{k}(x)\right)-\frac{1}{b-a} \int_{a}^{b} f(t) d t=\frac{1}{n !(b-a)} \int_{a}^{b}(x-t)^{n-1} k(t, x) f^{(n)}(t) d t
$$

where

$$
\begin{gathered}
F_{k}(x)=\frac{n-k}{k !} \frac{f^{(k-1)}(a)(x-a)^{k}-f^{(k-1)}(b)(x-b)^{k}}{b-a}, \\
k(t, x)= \begin{cases}t-a, & a \leq t \leq x \leq b, \\
t-b, & a \leq x<t \leq b .\end{cases}
\end{gathered}
$$


In fact, Milovanović and Pečarić have proved that

$$
K(n, \infty, x)=\frac{(x-a)^{n+1}+(b-x)^{n+1}}{n(n+1) !(b-a)},
$$

while Fink gave the following generalizations of this result.

Theorem 1.1. Let $f^{(n-1)}$ be absolutely continuous on $[a, b]$ and let $f^{(n)} \in L_{p}[a, b]$. Then inequality (1.2) holds with

$$
K(n, p, x)=\frac{\left[(x-a)^{n q+1}+(b-x)^{n q+1}\right]^{1 / q}}{n !(b-a)} B((n-1) q+1, q+1)^{1 / q},
$$

where $1<p \leq \infty, 1 / p+1 / q=1, B$ is the Beta function, and

$$
K(n, 1, x)=\frac{(n-1)^{n-1}}{n^{n} n !(b-a)} \max \left[(x-a)^{n},(b-x)^{n}\right] .
$$

Let $f:[a, b] \rightarrow \mathbb{R}$ be differentiable on $[a, b]$ and $f^{\prime}:[a, b] \rightarrow \mathbb{R}$ integrable on $[a, b]$. Then the Montgomery identity holds [9]:

$$
f(x)=\frac{1}{b-a} \int_{a}^{b} f(t) d t+\int_{a}^{b} P(x, t) f^{\prime}(t) d t
$$

where $P(x, t)$ is the Peano kernel defined by

$$
P(x, t)= \begin{cases}\frac{t-a}{b-a}, & a \leq t \leq x, \\ \frac{t-b}{b-a}, & x<t \leq b .\end{cases}
$$

Now, we suppose $w:[a, b] \rightarrow[0, \infty\rangle$ is some probability density function, that is, an integrable function satisfying $\int_{a}^{b} w(t) d t=1$, and $W(t)=\int_{a}^{t} w(x) d x$ for $t \in[a, b], W(t)=0$ for $t<a$, and $W(t)=1$ for $t>b$. The following identity (given by Pečarić in [12]) is the weighted generalization of the Montgomery identity:

$$
f(x)=\int_{a}^{b} w(t) f(t) d t+\int_{a}^{b} P_{w}(x, t) f^{\prime}(t) d t
$$

where the weighted Peano kernel is

$$
P_{w}(x, t)= \begin{cases}W(t), & a \leq t \leq x, \\ W(t)-1, & x<t \leq b .\end{cases}
$$


The aim of this paper is to give the extension of the weighted Montgomery identity (1.10) using identity (1.2), and further, obtain some new Ostrowski-type inequalities, as well as the generalizations of the estimations of the difference of two weighted integral means (generalizations of the results from $[1,3,7,11]$ ).

\section{The extension of Montgomery identity via Fink identity}

THEOREM 2.1. Let $f:[a, b] \rightarrow \mathbb{R}$ be such that $f^{(n-1)}$ is an absolutely continuous function on $[a, b]$ for some $n \geq 1$. If $w:[a, b] \rightarrow[0, \infty\rangle$ is some probability density function, then the following identity holds:

$$
\begin{aligned}
f(x)= & \int_{a}^{b} w(t) f(t) d t-\sum_{k=1}^{n-1} F_{k}(x)+\sum_{k=1}^{n-1} \int_{a}^{b} w(t) F_{k}(t) d t \\
& +\frac{1}{(n-1) !(b-a)} \int_{a}^{b}(x-y)^{n-1} k(y, x) f^{(n)}(y) d y \\
& -\frac{1}{(n-1) !(b-a)} \int_{a}^{b}\left(\int_{a}^{b} w(t)(t-y)^{n-1} k(y, t) d t\right) f^{(n)}(y) d y .
\end{aligned}
$$

Proof. We apply identity (1.3) with $f^{\prime}(t)$ :

$$
\begin{aligned}
f^{\prime}(t)= & -\sum_{k=1}^{n-1} \frac{n-k}{k !} \frac{f^{(k)}(a)(t-a)^{k}-f^{(k)}(b)(t-b)^{k}}{b-a}+n \frac{f(b)-f(a)}{b-a} \\
& +\frac{1}{(n-1) !(b-a)} \int_{a}^{b}(t-y)^{n-1} k(y, t) f^{(n+1)}(y) d y \\
= & -\sum_{k=0}^{n-1} \frac{n-k}{k !} \frac{f^{(k)}(a)(t-a)^{k}-f^{(k)}(b)(t-b)^{k}}{b-a} \\
& +\frac{1}{(n-1) !(b-a)} \int_{a}^{b}(t-y)^{n-1} k(y, t) f^{(n+1)}(y) d y .
\end{aligned}
$$

Now, by putting this formula in the weighted Montgomery identity (1.10), we obtain

$$
\begin{aligned}
f(x)= & \int_{a}^{b} w(t) f(t) d t \\
& -\sum_{k=0}^{n-1} \frac{n-k}{k !} \int_{a}^{b} P_{w}(x, t) \frac{f^{(k)}(a)(t-a)^{k}-f^{(k)}(b)(t-b)^{k}}{b-a} d t \\
& +\frac{1}{(n-1) !(b-a)} \int_{a}^{b} P_{w}(x, t)\left(\int_{a}^{b}(t-y)^{n-1} k(y, t) f^{(n+1)}(y) d y\right) d t .
\end{aligned}
$$


Further,

$$
\begin{gathered}
\int_{a}^{b} P_{w}(x, t) \frac{f^{(k)}(a)(t-a)^{k}-f^{(k)}(b)(t-b)^{k}}{b-a} d t \\
=\frac{f^{(k)}(a)(x-a)^{k+1}-f^{(k)}(b)(x-b)^{k+1}}{(b-a)(k+1)} \\
\quad-\int_{a}^{b} w(t) \frac{f^{(k)}(a)(t-a)^{k+1}-f^{(k)}(b)(t-b)^{k+1}}{(b-a)(k+1)} d t \\
\int_{a}^{b} P_{w}(x, t)(t-y)^{n-1} k(y, t) d t \\
=\frac{1}{n}(x-y)^{n} k(y, x)-\frac{1}{n} \int_{a}^{b} w(t)(t-y)^{n} k(y, t) d t .
\end{gathered}
$$

Now, if we replace $n$ with $n-1$, we will get (2.1). This identity is valid for $n-1 \geq 1$, that is, $n>1$.

Remark 2.2. We could also obtain identity (2.1) by applying identity (1.3) such that we multiply this identity by $w(x)$ and than integrate it to obtain

$$
\begin{aligned}
\int_{a}^{b} w(x) f(x) d x= & -\sum_{k=1}^{n-1} \int_{a}^{b} w(x) F_{k}(x) d x+\left(\int_{a}^{b} w(x) d x\right) \frac{n}{b-a} \int_{a}^{b} f(t) d t \\
& +\frac{1}{(n-1) !(b-a)} \int_{a}^{b}\left(\int_{a}^{b} w(x)(x-t)^{n-1} k(t, x) d x\right) f^{(n)}(t) d t .
\end{aligned}
$$

If we subtract this identity from (1.3) we will obtain (2.1).

Remark 2.3. In the special case, if we take $w(t)=1 /(b-a), t \in[a, b]$, we will have

$$
\begin{aligned}
\frac{1}{b-a} \sum_{k=1}^{n-1} \int_{a}^{b} F_{k}(t) d t & =\frac{1}{b-a} \sum_{k=1}^{n-1} \frac{n-k}{k !} \int_{a}^{b} \frac{f^{(k-1)}(a)(t-a)^{k}-f^{(k-1)}(b)(t-b)^{k}}{b-a} d t \\
& =\sum_{k=1}^{n-1} \frac{n-k}{(k+1) !}\left[f^{(k-1)}(a)(b-a)^{k-1}+f^{(k-1)}(b)(a-b)^{k-1}\right], \\
\frac{1}{b-a} \int_{a}^{b}(t-y)^{n-1} k(y, t) d t & =k(y, b) \frac{(b-y)^{n}}{n(b-a)}-k(y, a) \frac{(a-y)^{n}}{n(b-a)} \\
& =\frac{(y-a)(b-y)^{n}}{n(b-a)}-\frac{(y-b)(a-y)^{n}}{n(b-a)} .
\end{aligned}
$$


We denote

$$
I_{n}=\frac{1}{n !(b-a)^{2}} \int_{a}^{b}\left[(y-a)(b-y)^{n}-(y-b)(a-y)^{n}\right] f^{(n)}(y) d y .
$$

Then we have

$$
I_{n}=\frac{1}{n !(b-a)^{2}} \int_{a}^{b}\left[(a-y)^{n}-(b-y)^{n}\right] f^{(n-1)}(y) d y+I_{n-1}=J_{n}+I_{n-1},
$$

where

$$
I_{0}=\frac{1}{(b-a)^{2}} \int_{a}^{b}(b-a) f(y) d y=\frac{1}{b-a} \int_{a}^{b} f(y) d y
$$

Further,

$$
\begin{gathered}
J_{n}=\frac{1}{n !}\left[f^{(n-2)}(a)(b-a)^{n-2}+f^{(n-2)}(b)(a-b)^{n-2}\right]+J_{n-1}, \\
J_{1}=\frac{1}{(b-a)^{2}} \int_{a}^{b}(a-b) f(y) d y=-\frac{1}{b-a} \int_{a}^{b} f(y) d y .
\end{gathered}
$$

So,

$$
J_{n}=\sum_{k=1}^{n-1} \frac{1}{(k+1) !}\left[f^{(k-1)}(a)(b-a)^{k-1}+f^{(k-1)}(b)(a-b)^{k-1}\right]+J_{1},
$$

and then

$$
\begin{aligned}
I_{n}= & \sum_{m=2}^{n} J_{m}+n J_{1}+I_{0} \\
= & \sum_{k=1}^{n-1} \frac{n-k}{(k+1) !}\left[f^{(k-1)}(a)(b-a)^{k-1}+f^{(k-1)}(b)(a-b)^{k-1}\right] \\
& -\frac{n-1}{b-a} \int_{a}^{b} f(y) d y .
\end{aligned}
$$

Consequently, identity (2.1) reduces to identity (1.3). So we may regard it as a weighted Fink identity. 
Remark 2.4. Applying identity (2.1) with $x=a$ and $x=b$, we get

$$
\begin{aligned}
f(a)= & \int_{a}^{b} w(t) f(t) d t-\sum_{k=1}^{n-1} \frac{n-k}{k !} f^{(k-1)}(b)(a-b)^{k-1}+\sum_{k=1}^{n-1} \int_{a}^{b} w(t) F_{k}(t) d t \\
& +\frac{1}{(n-1) !(b-a)} \int_{a}^{b}(a-y)^{n-1}(y-b) f^{(n)}(y) d y \\
& -\frac{1}{(n-1) !(b-a)} \int_{a}^{b}\left(\int_{a}^{b} w(t)(t-y)^{n-1} k(y, t) d t\right) f^{(n)}(y) d y, \\
f(b)= & \int_{a}^{b} w(t) f(t) d t-\sum_{k=1}^{n-1} \frac{n-k}{k !} f^{(k-1)}(a)(b-a)^{k-1}+\sum_{k=1}^{n-1} \int_{a}^{b} w(t) F_{k}(t) d t \\
& +\frac{1}{(n-1) !(b-a)} \int_{a}^{b}(b-y)^{n-1}(y-a) f^{(n)}(y) d y \\
& -\frac{1}{(n-1) !(b-a)} \int_{a}^{b}\left(\int_{a}^{b} w(t)(t-y)^{n-1} k(y, t) d t\right) f^{(n)}(y) d y .
\end{aligned}
$$

So, we get the generalized trapezoid identity

$$
\begin{aligned}
\frac{1}{2}[f(a)+f(b)]= & \int_{a}^{b} w(t) f(t) d t+\sum_{k=1}^{n-1} \int_{a}^{b} w(t) F_{k}(t) d t \\
& -\frac{1}{2} \sum_{k=1}^{n-1} \frac{n-k}{k !}\left[f^{(k-1)}(a)(b-a)^{k-1}+f^{(k-1)}(b)(a-b)^{k-1}\right] \\
& +\frac{1}{2(n-1) !(b-a)} \int_{a}^{b}\left[(a-y)^{n-1}(y-b)+(b-y)^{n-1}(y-a)\right] f^{(n)}(y) d y \\
& -\frac{1}{(n-1) !(b-a)} \int_{a}^{b}\left(\int_{a}^{b} w(t)(t-y)^{n-1} k(y, t) d t\right) f^{(n)}(y) d y .
\end{aligned}
$$

Similarly, applying identity (2.1) with $x=(a+b) / 2$, we get

$$
\begin{aligned}
f\left(\frac{a+b}{2}\right)= & \int_{a}^{b} w(t) f(t) d t+\sum_{k=1}^{n-1} \int_{a}^{b} w(t) F_{k}(t) d t \\
& -\sum_{k=1}^{n-1} \frac{n-k}{2^{k} k !}\left[f^{(k-1)}(a)(b-a)^{k-1}+f^{(k-1)}(b)(a-b)^{k-1}\right] \\
& +\frac{1}{(n-1) !(b-a)} \int_{a}^{b}\left(\frac{a+b}{2}-y\right)^{n-1} k\left(y, \frac{a+b}{2}\right) f^{(n)}(y) d y \\
& -\frac{1}{(n-1) !(b-a)} \int_{a}^{b}\left(\int_{a}^{b} w(t)(t-y)^{n-1} k(y, t) d t\right) f^{(n)}(y) d y .
\end{aligned}
$$

We can regard this as the second Euler-Maclaurin formula (the generalized midpoint identity). 


\section{Ostrowski-type inequalities}

We denote, for $n \geq 2$,

$$
T_{w, n}(x)=\sum_{k=1}^{n-1} F_{k}(x)-\sum_{k=1}^{n-1} \int_{a}^{b} w(t) F_{k}(t) d t .
$$

Theorem 3.1. Assume $(p, q)$ is a pair of conjugate exponents, that is, $1 \leq p, q \leq \infty, 1 / p+$ $1 / q=1$. Let $\left|f^{(n)}\right|^{p}:[a, b] \rightarrow \mathbb{R}$ be an R-integrable function for some $n>1$. Then, for $x \in$ $[a, b]$, the following inequality holds:

$$
\begin{aligned}
\mid f(x) & -\int_{a}^{b} w(t) f(t) d t+T_{w, n}(x) \mid \\
& \leq \frac{1}{(n-2) !(b-a)}\left(\int_{a}^{b}\left|\int_{a}^{b} P_{w}(x, t)(t-y)^{n-2} k(y, t) d t\right|^{q} d y\right)^{1 / q}\left\|f^{(n)}\right\|_{p} .
\end{aligned}
$$

The constant $(1 /(n-2) !(b-a))\left(\int_{a}^{b}\left|\int_{a}^{b} P_{w}(x, t)(t-y)^{n-2} k(y, t) d t\right|^{q} d y\right)^{1 / q}$ is sharp for $1<$ $p \leq \infty$ and is the best possible for $p=1$.

Proof. From Theorem 2.1 we have

$$
(x-y)^{n-1} k(y, x)-\int_{a}^{b} w(t)(t-y)^{n-1} k(y, t) d t=(n-1) \int_{a}^{b} P_{w}(x, t)(t-y)^{n-2} k(y, t) d t .
$$

We denote $C_{1}(y)=(1 /(n-2) !(b-a)) \int_{a}^{b} P_{w}(x, t)(t-y)^{n-2} k(y, t) d t$. We use identity $(2.1)$ and apply the Hölder inequality to obtain

$$
\begin{aligned}
\mid f(x) & -\int_{a}^{b} w(t) f(t) d t+T_{w, n}(x) \mid \\
= & \left|\int_{a}^{b} C_{1}(y) f^{(n)}(y) d y\right| \leq\left(\int_{a}^{b}\left|C_{1}(y)\right|^{q} d y\right)^{1 / q}\left\|f^{(n)}\right\|_{p} .
\end{aligned}
$$

For the proof of the sharpness of the constant $\left(\int_{a}^{b}\left|C_{1}(y)\right|^{q} d y\right)^{1 / q}$, we will find a function $f$ for which the equality in (3.2) is obtained.

For $1<p<\infty$, take $f$ to be such that

$$
f^{(n)}(y)=\operatorname{sgn} C_{1}(y) \cdot\left|C_{1}(y)\right|^{1 /(p-1)} .
$$

For $p=\infty$, take

$$
f^{(n)}(y)=\operatorname{sgn} C_{1}(y) .
$$


74 The extension of Montgomery identity

For $p=1$, we will prove that

$$
\left|\int_{a}^{b} C_{1}(y) f^{(n)}(y) d t\right| \leq \max _{y \in[a, b]}\left|C_{1}(y)\right|\left(\int_{a}^{b}\left|f^{(n)}(y)\right| d y\right)
$$

is the best possible inequality. Suppose that $\left|C_{1}(y)\right|$ attains its maximum at $y_{0} \in[a, b]$. First we assume that $C_{1}\left(y_{0}\right)>0$. For $\varepsilon$ small enough, define $f_{\varepsilon}(y)$ by

$$
f_{\varepsilon}(y)= \begin{cases}0, & a \leq y \leq y_{0}, \\ \frac{1}{\varepsilon n !}\left(y-y_{0}\right)^{n}, & y_{0} \leq y \leq y_{0}+\varepsilon \\ \frac{1}{n !}\left(y-y_{0}\right)^{n-1}, & y_{0}+\varepsilon \leq y \leq b .\end{cases}
$$

Then, for $\varepsilon$ small enough,

$$
\left|\int_{a}^{b} C_{1}(y) f^{(n)}(y) d y\right|=\left|\int_{y_{0}}^{y_{0}+\varepsilon} C_{1}(y) \frac{1}{\varepsilon} d y\right|=\frac{1}{\varepsilon} \int_{y_{0}}^{y_{0}+\varepsilon} C_{1}(y) d y .
$$

Now, from inequality (3.7) we have

$$
\frac{1}{\varepsilon} \int_{y_{0}}^{y_{0}+\varepsilon} C_{1}(y) d y \leq C_{1}\left(y_{0}\right) \int_{y_{0}}^{y_{0}+\varepsilon} \frac{1}{\varepsilon} d y=C_{1}\left(y_{0}\right) .
$$

Since

$$
\lim _{\varepsilon \rightarrow 0} \frac{1}{\varepsilon} \int_{y_{0}}^{y_{0}+\varepsilon} C_{1}(y) d y=C_{1}\left(y_{0}\right)
$$

the statement follows. In case $C_{1}\left(y_{0}\right)<0$, we take

$$
f_{\varepsilon}(y)= \begin{cases}\frac{1}{n !}\left(y-y_{0}-\varepsilon\right)^{n-1}, & a \leq y \leq y_{0}, \\ -\frac{1}{\varepsilon n !}\left(y-y_{0}-\varepsilon\right)^{n}, & y_{0} \leq y \leq y_{0}+\varepsilon, \\ 0, & y_{0}+\varepsilon \leq y \leq b,\end{cases}
$$

and the rest of the proof is the same as above. 
Remark 3.2. For $w(t)=1 /(b-a), n=2$, and $q=1$ in Theorem 3.1, we get

$$
\begin{aligned}
\mid f(x) & -\frac{1}{b-a} \int_{a}^{b} f(t) d t-\left(x-\frac{a+b}{2}\right)(f(b)-f(a)) \mid \\
& \leq \frac{1}{b-a}\left(\int_{a}^{b}\left|(x-y) k(y, x)-\frac{1}{b-a} \int_{a}^{b}(t-y) k(y, t) d t\right| d y\right)\left\|f^{\prime \prime}\right\|_{\infty} \\
& =\frac{1}{b-a}\left(\int_{a}^{b}\left|(x-y) k(y, x)-\frac{(y-a)(b-y)}{2}\right| d y\right)\left\|f^{\prime \prime}\right\|_{\infty} \\
& =\frac{1}{2(b-a)}\left(\int_{a}^{x}|(y-a)(2 x-y-b)| d y+\int_{x}^{b}|(b-y)(-2 x+y+a)| d y\right)\left\|f^{\prime \prime}\right\|_{\infty} \\
& =\left(\frac{4}{3} \delta^{3}(x)-\frac{1}{2} \delta^{2}(x)+\frac{1}{24}\right)\left\|f^{\prime \prime}\right\|_{\infty},
\end{aligned}
$$

where $\delta(x)=|x-(a+b) / 2|$.

If instead of $q=1(p=\infty)$ we put $p=1$, then, similarly we have

$$
\begin{aligned}
\mid f(x) & -\frac{1}{b-a} \int_{a}^{b} f(t) d t-\left(x-\frac{a+b}{2}\right)(f(b)-f(a)) \mid \\
& \leq \frac{1}{2(b-a)} \max \left\{\max _{y \in[a, x]}|(y-a)(2 x-y-b)|, \max _{y \in[x, b]}|(b-y)(-2 x+y+a)|\right\}|| f^{\prime \prime} \|_{1} \\
& =\frac{1}{4}\left[\frac{1}{4}+\left|\frac{1}{4}-2\left(x-\frac{a+b}{2}\right)^{2}\right|\right]\left\|f^{\prime \prime}\right\|_{1} .
\end{aligned}
$$

These two inequalities are proved in [5].

Corollary 3.3. Suppose that all the assumptions of Theorem 3.1 hold. Then the following inequality holds:

$$
\begin{aligned}
& \left|f(x)-\int_{a}^{b} w(t) f(t) d t+T_{w, n}(x)\right| \\
& \quad \leq \frac{1}{(n-1) !(b-a)}\left(\int_{a}^{b}\left[(b-y)(y-a)^{n-1}+(y-a)(b-y)^{n-1}\right]^{q} d y\right)^{1 / q}\left\|f^{(n)}\right\|_{p} .
\end{aligned}
$$


76 The extension of Montgomery identity

Proof. Since $0 \leq W(t) \leq 1, t \in[a, b]$, so $\left|P_{w}(x, t)\right| \leq 1$. Then, for every $y \in[a, b]$, we have

$$
\begin{aligned}
& \left|\int_{a}^{b} P_{w}(x, t)(t-y)^{n-2} k(y, t) d t\right| \\
& \quad \leq \int_{a}^{b}\left|P_{w}(x, t)\right|\left|(t-y)^{n-2} k(y, t)\right| d t \\
& \quad \leq \int_{a}^{b}\left|(t-y)^{n-2} k(y, t)\right| d t \\
& \quad=\left[\int_{a}^{y}(y-t)^{n-2}(b-y) d t+\int_{y}^{b}(t-y)^{n-2}(y-a) d t\right] \\
& \quad=\frac{1}{n-1}\left[(b-y)(y-a)^{n-1}+(y-a)(b-y)^{n-1}\right] .
\end{aligned}
$$

So,

$$
\begin{aligned}
& \left(\int_{a}^{b}\left|\int_{a}^{b} P_{w}(x, t)(t-y)^{n-1} k(y, t) d t\right|^{q} d y\right)^{1 / q} \\
& \quad \leq \frac{1}{n-1}\left(\int_{a}^{b}\left[(b-y)(y-a)^{n-1}+(y-a)(b-y)^{n-1}\right]^{q} d t\right)^{1 / q}
\end{aligned}
$$

and, by applying (3.2), the inequality is proved.

Remark 3.4. Inequality (3.15) reduces to the following: for $n=2$,

$$
\begin{aligned}
\mid f(x) & -\int_{a}^{b} w(t) f(t) d t+T_{w, 2}(x) \mid \\
& \leq \frac{2}{b-a}\left(\int_{a}^{b}(b-y)^{q}(y-a)^{q} d y\right)^{1 / q}\left\|f^{\prime \prime}\right\|_{p} \\
& =2(b-a)^{(q+1) / q}\left(\int_{0}^{1}(1-s)^{q} s^{q} d s\right)^{1 / q}\left\|f^{\prime \prime}\right\|_{p} \\
& =2(b-a)^{(q+1) / q} B(q+1, q+1)^{1 / q}\left\|f^{\prime \prime}\right\|_{p} .
\end{aligned}
$$

For $n=3$,

$$
\begin{aligned}
\mid f(x) & -\int_{a}^{b} w(t) f(t) d t+T_{w, 3}(x) \mid \\
& \leq \frac{1}{2(b-a)}\left(\int_{a}^{b}(b-y)^{q}(y-a)^{q}(b-a)^{q} d y\right)^{1 / q}\left\|f^{\prime \prime}\right\|_{p} \\
& =\frac{1}{2}(b-a)^{(2 q+1) / q} B(q+1, q+1)^{1 / q}\left\|f^{\prime \prime \prime}\right\|_{p} .
\end{aligned}
$$


Remark 3.5. If we use the identities (2.14) and (2.15) for $n=2$ and $w(t)=1 /(b-a)$, $t \in[a, b]$, and then apply the Hölder inequality with $p=\infty, q=1$, we will obtain

$$
\begin{aligned}
& \left|\frac{f(a)+f(b)}{2}-\frac{1}{b-a} \int_{a}^{b} f(t) d t\right| \leq \frac{(b-a)^{2}}{12}\left\|f^{\prime \prime}\right\|_{\infty}, \\
& \left|f\left(\frac{a+b}{2}\right)-\frac{1}{b-a} \int_{a}^{b} f(t) d t\right| \leq \frac{(b-a)^{2}}{24}\left\|f^{\prime \prime}\right\|_{\infty} .
\end{aligned}
$$

By doing the same for $n=3$, we have

$$
\begin{aligned}
& \left|\frac{f(a)+f(b)}{2}-\frac{1}{b-a} \int_{a}^{b} f(t) d t-\frac{b-a}{12}\left[f^{\prime}(b)-f^{\prime}(a)\right]\right| \leq \frac{(b-a)^{3}}{192}\left\|f^{\prime \prime \prime}\right\|_{\infty}, \\
& \left|f\left(\frac{a+b}{2}\right)-\frac{1}{b-a} \int_{a}^{b} f(t) d t+\frac{b-a}{24}\left[f^{\prime}(b)-f^{\prime}(a)\right]\right| \leq \frac{(b-a)^{3}}{192}\left\|f^{\prime \prime \prime}\right\|_{\infty} .
\end{aligned}
$$

The first two inequalities were obtained in [4] and the last two in [2].

\section{Estimations of the difference of two weighted integral means}

In this section, we will denote, for $n>1$,

$$
T_{w, n}^{[a, b]}(x)=\sum_{k=1}^{n-1} F_{k}^{[a, b]}(x)-\sum_{k=1}^{n-1} \int_{a}^{b} w(t) F_{k}^{[a, b]}(t) d t
$$

for a function $f:[a, b] \rightarrow \mathbb{R}$ such that $f^{(n-1)}$ is an absolutely continuous function on $[a, b]$.

The following results are generalizations of the results from [3] in two cases. The first case is when $[c, d] \subseteq[a, b]$ and the second is when $[a, b] \cap[c, d]=[c, b]$. Other two possible cases, when $[a, b] \cap[c, d] \neq \varnothing([a, b] \subset[c, d]$ and $[a, b] \cap[c, d]=[a, d])$ are simply got by change $a \leftrightarrow c, b \leftrightarrow d$.

Theorem 4.1. Let $f:[a, b] \cup[c, d] \rightarrow \mathbb{R}$ be such that $f^{(n-1)}$ is an absolutely continuous function on $[a, b]$ for some $n>1$, and let $w:[a, b] \rightarrow[0, \infty\rangle$ and $u:[c, d] \rightarrow[0, \infty\rangle$ be some probability density functions. Then, if $[a, b] \cap[c, d] \neq \varnothing$ and $x \in[a, b] \cap[c, d]$,

$$
\int_{a}^{b} w(t) f(t) d t-\int_{c}^{d} u(t) f(t) d t-T_{w, n}^{[a, b]}(x)+T_{u, n}^{[c, d]}(x)=\int_{\min \{a, c\}}^{\max \{b, d\}} K_{n}(x, y) f^{(n)}(y) d y,
$$


where, in case $[c, d] \subseteq[a, b]$,

$$
K_{n}(x, y)= \begin{cases}\frac{-1}{(n-2) !(b-a)}\left[\int_{a}^{b} P_{w}(x, t)(t-y)^{n-2} k^{[a, b]}(y, t) d t\right], & y \in[a, c], \\ \frac{-1}{(n-2) !(b-a)}\left[\int_{a}^{b} P_{w}(x, t)(t-y)^{n-2} k^{[a, b]}(y, t) d t\right] & \\ +\frac{1}{(n-2) !(d-c)}\left[\int_{c}^{d} P_{u}(x, t)(t-y)^{n-2} k^{[c, d]}(y, t) d t\right], & y \in\langle c, d], \\ \frac{-1}{(n-2) !(b-a)}\left[\int_{a}^{b} P_{w}(x, t)(t-y)^{n-2} k^{[a, b]}(y, t) d t\right], & y \in\langle d, b],\end{cases}
$$

and in case $[a, b] \cap[c, d]=[c, b]$,

$$
K_{n}(x, y)= \begin{cases}\frac{-1}{(n-2) !(b-a)}\left[\int_{a}^{b} P_{w}(x, t)(t-y)^{n-2} k^{[a, b]}(y, t) d t\right], & y \in[a, c], \\ \frac{-1}{(n-2) !(b-a)}\left[\int_{a}^{b} P_{w}(t)(t-y)^{n-2} k^{[a, b]}(y, t) d t\right] & \\ +\frac{1}{(n-2) !(d-c)}\left[\int_{c}^{d} P_{u}(t)(t-y)^{n-2} k^{[c, d]}(y, t) d t\right], & y \in\langle c, b], \\ \frac{1}{(n-2) !(d-c)}\left[\int_{c}^{d} P_{u}(t)(t-y)^{n-2} k^{[c, d]}(y, t) d t\right], & y \in\langle b, d] .\end{cases}
$$

Proof. We subtract identity (2.1) for intervals $[a, b]$ and $[c, d]$ to get formula (4.2).

Theorem 4.2. Assume $(p, q)$ is a pair of conjugate exponents, that is, $1 \leq p, q \leq \infty, 1 / p+$ $1 / q=1$. Let $\left|f^{(n)}\right|^{p}:[a, b] \rightarrow \mathbb{R}$ be an $R$-integrable function for some $n>1$. Then

$$
\begin{gathered}
\left|\int_{a}^{b} w(t) f(t) d t-\int_{c}^{d} u(t) f(t) d t-T_{w, n}^{[a, b]}(x)+T_{u, n}^{[c, d]}(x)\right| \\
\quad \leq\left(\int_{\min \{a, c\}}^{\max \{b, d\}}\left|K_{n}(x, y)\right|^{q} d y\right)^{1 / q}\left\|f^{(n)}\right\|_{p}
\end{gathered}
$$

for every $x \in[a, b] \cap[c, d]$. The constant $\left(\int_{\min \{a, c\}}^{\max \{b, d\}}\left|K_{n}(x, y)\right|^{q} d y\right)^{1 / q}$ in inequality (4.5) is sharp for $1<p \leq \infty$ and is the best possible for $p=1$.

Proof. Use identity (4.2) and apply the Hölder inequality to obtain

$$
\begin{aligned}
& \left|\int_{a}^{b} w(t) f(t) d t-\int_{c}^{d} u(t) f(t) d t-T_{w, n}^{[a, b]}(x)+T_{u, n}^{[c, d]}(x)\right| \\
& \quad \leq \int_{\min \{a, c\}}^{\max \{b, d\}}\left|K_{n}(x, y)\right|\left|f^{(n)}(y)\right| d y \leq\left(\int_{\min \{a, c\}}^{\max \{b, d\}}\left|K_{n}(x, y)\right|^{q} d y\right)^{1 / q}\left\|f^{(n)}\right\|_{p},
\end{aligned}
$$


which proves inequality (4.5). The proofs for sharpness and best possibility are as in Theorem 3.1.

Corollary 4.3. Suppose that all the assumptions of Theorem 4.2 hold. Then, for $x \in[a, b] \cap$ $[c, d]$

$$
\begin{aligned}
& \left|\int_{a}^{b} w(t) f(t) d t-\int_{c}^{d} u(t) f(t) d t-T_{w, n}^{[a, b]}(x)+T_{u, n}^{[c, d]}(x)\right| \\
& \quad \leq \frac{2}{(n-1) !}\left(\int_{a}^{\max \{b, d\}}\left|(y-a)^{n-1}+(\max \{b, d\}-y)^{n-1}\right|^{q} d y\right)^{1 / q}\left\|f^{(n)}\right\|_{p} .
\end{aligned}
$$

Proof. We have

$$
K_{n}(x, y)=\frac{-1}{(n-2) !} \int_{\min \{a, c\}}^{\max \{b, d\}}\left[P_{w}(x, t) \frac{k^{[a, b]}(y, t)}{b-a}-P_{u}(x, t) \frac{k^{[c, d]}(y, t)}{d-c}\right](t-y)^{n-2} d t
$$

because $P_{w}(x, t)=0$, for $x \notin[a, b]$ and $P_{u}(x, t)=0$, for $x \notin[c, d]$. Since

$$
-1 \leq P_{w}(x, t), P_{u}(x, t), \frac{k^{[a, b]}(y, t)}{b-a}, \frac{k^{[c, d]}(y, t)}{d-c} \leq 1,
$$

we get

$$
\left|P_{w}(x, t) \frac{k^{[a, b]}(y, t)}{b-a}-P_{u}(x, t) \frac{k^{[c, d]}(y, t)}{d-c}\right| \leq 2
$$

and then we have

$$
\left|K_{n}(x, y)\right| \leq \frac{2}{(n-2) !} \int_{a}^{\max \{b, d\}}|t-y|^{n-2} d t=\frac{2\left((y-a)^{n-1}+(\max \{b, d\}-y)^{n-1}\right)}{(n-1) !}
$$

\section{References}

[1] A. A. Aljinović, J. Pečarić, and I. Perić, Estimates of the difference between two weighted integral means via weighted Montgomery identity, Math. Inequal. Appl. 7 (2004), no. 3, 315-336.

[2] A. A. Aljinović and J. Pečarić, The weighted Euler identity, to appear in Math. Inequal. Appl.

[3] N. S. Barnett, P. Cerone, S. S. Dragomir, and A. M. Fink, Comparing two integral means for absolutely continuous mappings whose derivatives are in $L_{\infty}[a, b]$ and applications, Comput. Math. Appl. 44 (2002), no. 1-2, 241-251.

[4] Lj. Dedić, M. Matić, and J. Pečarić, On generalizations of Ostrowski inequality via some Eulertype identities, Math. Inequal. Appl. 3 (2000), no. 3, 337-353.

[5] Lj. Dedić, M. Matić, J. Pečarić, and A. Vukelić, On generalizations of Ostrowski inequality via Euler harmonic identities, J. Inequal. Appl. 7 (2002), no. 6, 787-805.

[6] A. M. Fink, Bounds on the deviation of a function from its averages, Czechoslovak Math. J. 42(117) (1992), no. 2, 289-310. 
[7] M. Matić and J. Pečarić, Two-point Ostrowski inequality, Math. Inequal. Appl. 4 (2001), no. 2, 215-221.

[8] G. V. Milovanović and J. E. Pečarić, On generalization of the inequality of A. Ostrowski and some related applications, Univ. Beograd. Publ. Elektrotehn. Fak. Ser. Mat. Fiz. (1976), no. 544576, 155-158.

[9] D. S. Mitrinović, J. E. Pečarić, and A. M. Fink, Inequalities for Functions and Their Integral and Derivatives, Kluwer Academic Publishers, Dordrecht, 1994.

[10] A. Ostrowski, Über die Absolutabweichung einer differentiebaren funktion von ihren integralmittelwert, Comment. Math. Helv. 10 (1938), 226-227 (German).

[11] J. Pečarić, I. Perić, and A. Vukelić, Estimations of the difference of two integral means via Eulertype identities, Math. Inequal. Appl. 7 (2004), no. 3, 365-378.

[12] J. E. Pečarić, On the Čebyšev inequality, Bul. Şti. Tehn. Inst. Politehn. “Traian Vuia” Timişoara 25(39) (1980), no. 1, 5-9.

A. Aglić Aljinović: Department of Applied Mathematics, Faculty of Electrical Engineering and Computing, University of Zagreb, Unska 3, 10000 Zagreb, Croatia

E-mail address: andrea@zpm.fer.hr

J. Pečarić: Department of Mathematics, Faculty of Textile Technology, University of Zagreb, Pierottijeva 6, 10000 Zagreb, Croatia

E-mail address: pecaric@hazu.hr

A. Vukelić: Mathematics Department, Faculty of Food Technology and Biotechnology, University of Zagreb, Pierottijeva 6, 10000 Zagreb, Croatia

E-mail address: avukelic@pbf.hr 